\title{
SPI-ACS Timing: Cross-Check of Onboard Clocks
}

\author{
Xiao-Ling Zhang*, Arne Rau, Andreas von Kienlin \\ Max-Planck Institute for Extraterrestrial Physics \\ Giessenbachstr. 1, D-85748, Garching, Germany \\ E-mail: zhangx@mpe.mpg.de, arau@mpe.mpg.de, azk@mpe.mpg.de \\ Kevin Hurley \\ University of California Space Sciences Laboratory \\ 7 Gauss Way, Berkeley, CA, 94720-7450 \\ E-mail: khurley@ssl.berkeley.edu
}

The SPI-ACS is an important component in the InterPlanetary Network, which utilizes Gammaray satellites to localize Gamma Ray Bursts through triangulation. For this purpose, it is important to know the accuracy of the ACS onboard clock. The SPI germanium detectors use a different clock, therefore we can calibrate the ACS clock with the SPI detector clock. In this study we use high energy cosmic-ray events which cause spikes in the ACS and at the same time saturate one or more SPI germanium detectors. We collect such events from more than 900 revolutions, obtain the distribution of the time difference between ACS spikes and corresponding SPI saturations, and compare the result with a Monte-Carlo simulation. We conclude that the ACS on board clock does not have significant drift with respect to the SPI detector clock.

8th INTEGRAL Workshop "The Restless Gamma-ray Universe"

September 27-30 2010

Dublin Castle, Dublin, Ireland

* Speaker. 


\section{Introduction}

The Anti-Coincidence Shield (ACS) of the Spectrometer onboard INTEGRAL (SPI) (Fig. 1) consists of $512 \mathrm{~kg}$ BGO crystals, sensitive to energies above $80 \mathrm{keV}$, with an effective area of about $5000 \mathrm{~cm}^{2}$ at $100 \mathrm{keV}[1,2]$. The BGO crystals surround the germanium detectors from the side and the bottom, which, together with its big effective area, makes ACS a sensitive and almost omni-directional gamma-ray burst (GRB) detector. It also provides high timing resolution at 50ms. However, it has no energy resolution, and almost no spatial information for GRBs.

Since the launch of INTEGRAL on 17th of October, 2002, it has been part of the In-

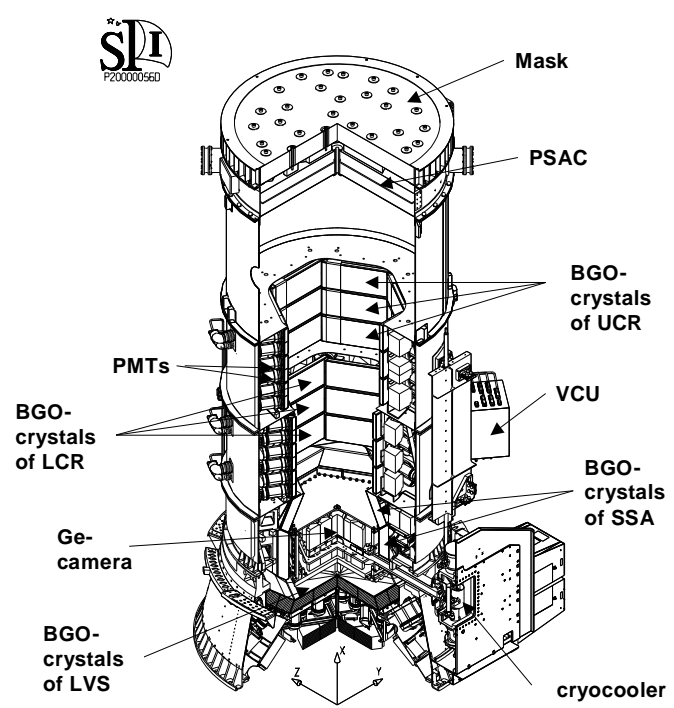

Figure 1: The Spectrometer onboard INTEGRAL (SPI)

terPlanetary Network (IPN)[3, 4, 5]. The IPN uses triangulation from the time recorded by different satellites to locate gamma-ray bursts. The highly elliptical orbit of INTEGRAL makes it a unique component in the IPN system, especially in the mini-network consists of earth-orbiting satellites[5]. For this purpose, the timing accuracy is important; since the ACS clock is not synchronized during the whole mission, we need to know the accuracy of the clock. The SPI germanium detectors use a different clock. It is possible to use high energy cosmic-ray events - which leave their traces in both the ACS and the germanium detectors at the same time - to cross check the two clocks.

\section{Saturation Events}

Sometimes a very short spike (one or two bins) in ACS count rate and the saturation of one or more germanium detectors can happen simultaneously [6] (Fig. 3), when, for instance, an energetic cosmic-ray particle deposits its energy partly in the ACS, and partly in one or more germanium detectors. Here an ACS spike accompanied by germanium detector saturation is called a saturation event. As the ACS spike and the ger-

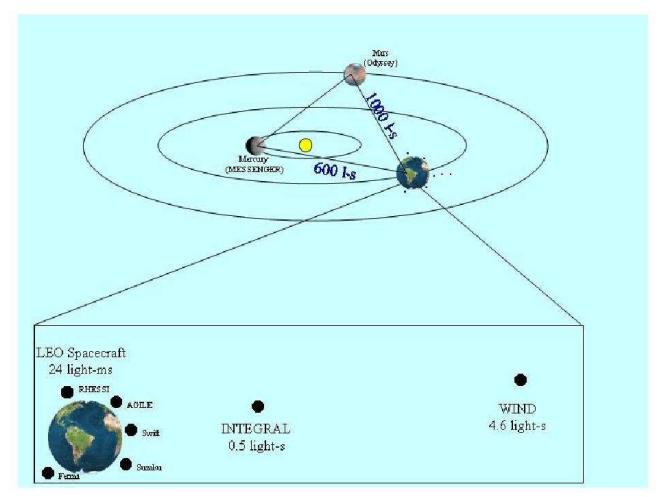

Figure 2: Current configuration of the Inter Planetary Network (IPN) manium saturation can be considered happening at the same time, but being recorded at slightly different times by the two clocks, we can use these events to cross check the two clocks. 

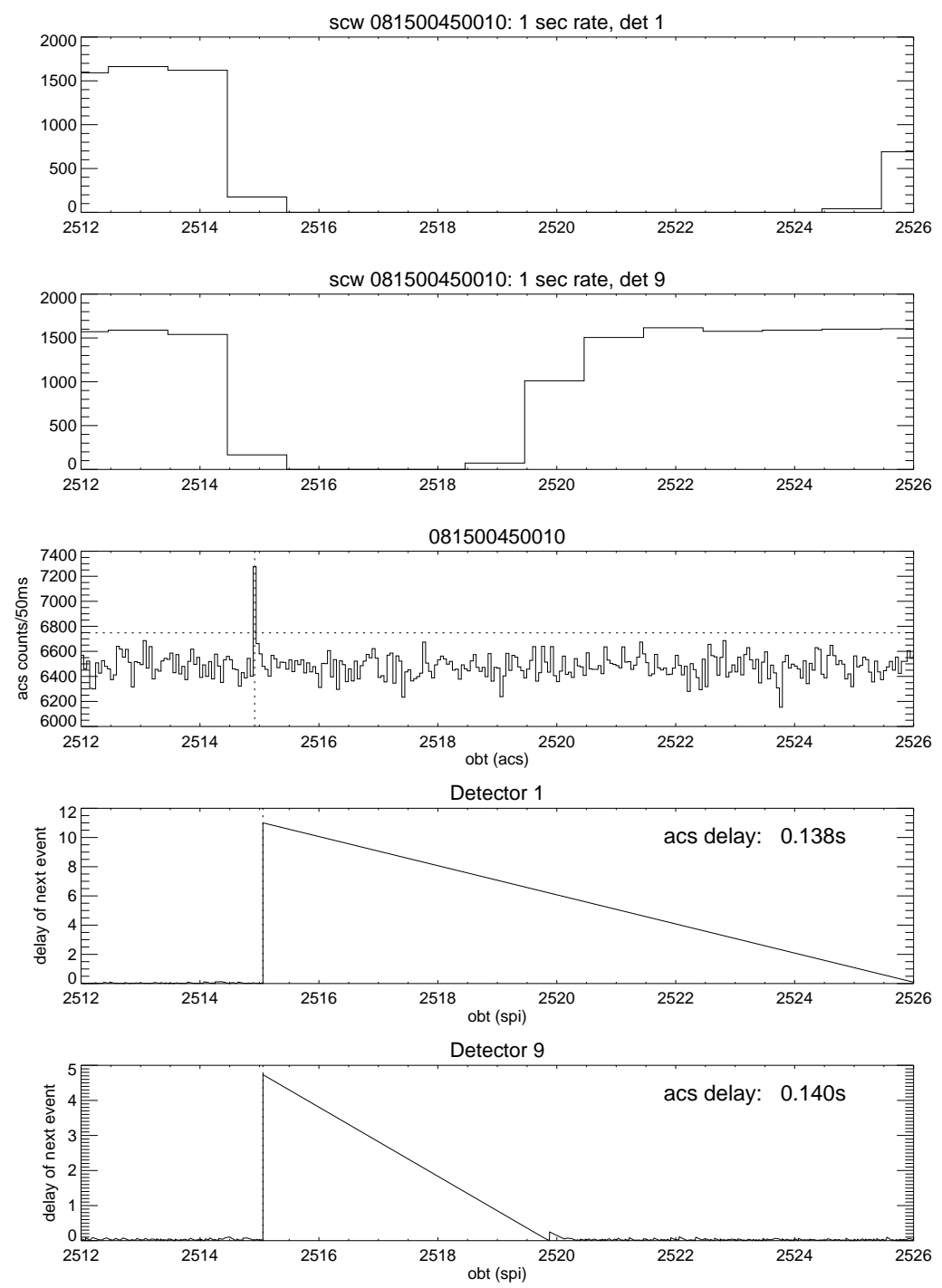

Figure 3: An example of saturation events, which happened in science window (ScW) 081500450010. In all panels the time is the corresponding onboard time (OBT) in seconds, shifted by the OBT of the first Germanium event in this science window. The top two panels are 1-second count rate (total events, including vetoed ones) of detectors 1 and 9, respectively. The middle panel shows an almost simultaneous ACS spike. Bottom two panels show for each photon event the time delay of the next event, in seconds. Normally this should be in the order of $20 \mathrm{~ms}$. The long time delay of the next event indicates that the detector has been flooded by the energetic event and is not able to register normal photon events. Here acs delay is the same as ACS time lag in text.

In this study we collect saturation events longer than 1 second in the 8 year mission of INTEGRAL, and compare the times recorded by the two clocks. As the germanium detector clock's timing resolution is $0.102 \mathrm{~ms}$, and the ACS time bin size is $50 \mathrm{~ms}$, we would expect that the time difference between the two times be uniformly distributed between $-25 \mathrm{~ms}$ and $25 \mathrm{~ms}$, if there is no drifting apart of the two clocks. 


\section{ACS Time Lag Distribution}

When comparing the times in saturation events, the OBT time of the last normal germanium event spi_time, recorded by the SPI germanium detector clock, is assumed to be the time of the detector saturation, and the time of the ACS spike acs_time is the time bin center, recorded by the ACS clock. Thus ACS time lag $\equiv$ spi_time - acs_time.
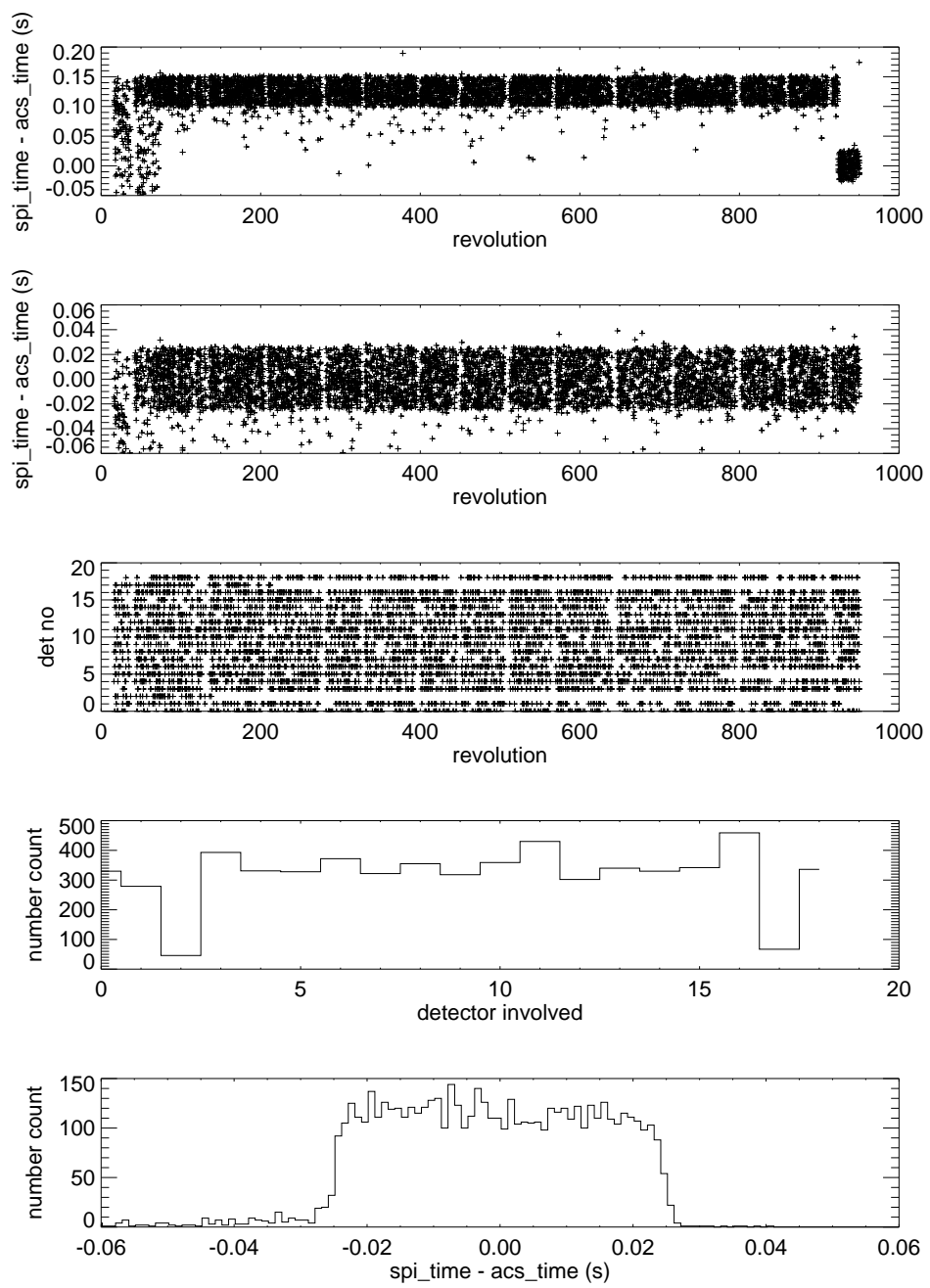

Figure 4: ACS time lag distribution. From top to bottom: 1, ACS time lag vs. revolution number. 2, same as panel 1, but the ACS time has been shifted by 0.125 second for revolutions earlier than 925. 3, ID of saturated detectors. No dependence on detectors. The failed detectors are easily seen. 4, Detector ID distribution. 5 (bottom), ACS time-lag distribution, bin-size $1 \mathrm{~ms}$. ACS time-lag has been shifted by $125 \mathrm{~ms}$ for revolutions before 925 .

In order to search for saturation events, we look for zero bins in the 1-second SPI rate for all (time-tagged) events, in the SPI house-keeping file spi_science_hk.fits (Fig. 3, top two panels), obtain spi_time from the normal SPI events from file spi_oper.fits. If there is an ACS spike ( $3 \sigma$ higher than mean value) close to spi_time, calculate the ACS Time Lag. In the rare 
cases that there are more than one ACS bins in the spike, use the highest one. If there are more than one saturated detectors, use the one with the smallest time difference with the ACS spike.

We have collected 6039 such events from revolution 14 to 950 with the above criteria. The result is shown in Fig. 4. The ACS time lag is uniformly distributed throughout the mission, except for the very early stage for unknown reason, and the very latest period; apparently from revolution 925 the ACS OBT has been corrected (offset by 0.125 second) as in the revision 3 data from ISDC, to take care of the known difference between the two clocks[7]. After shifting by 0.125 second for the early revolutions (before 925), the ACS time lag distribution is rather uniform between $25 \mathrm{~ms}$ and $25 \mathrm{~ms}$, which is because of the finite time bin of ACS. The rising and the falling edges characterize the possible drift between the ACS clock and SPI germanium detector clock.

\section{Monte-Carlo Simulation}

We performed a Monte-Carlo simulation to check if there is any drift between the clocks. The model is a $50 \mathrm{~ms}$ wide top-hat distribution centered at $o f f s e t$, and offset by a value following an exponential distribution with e-folding parameter tau (ms). The latter part is to account for any possible discrepancy caused by either the drift of the clock, or the time between the last SPI detector event and the energetic cosmic-ray event. The sample size is 10 times larger than that of the collected events, to reduce noise.
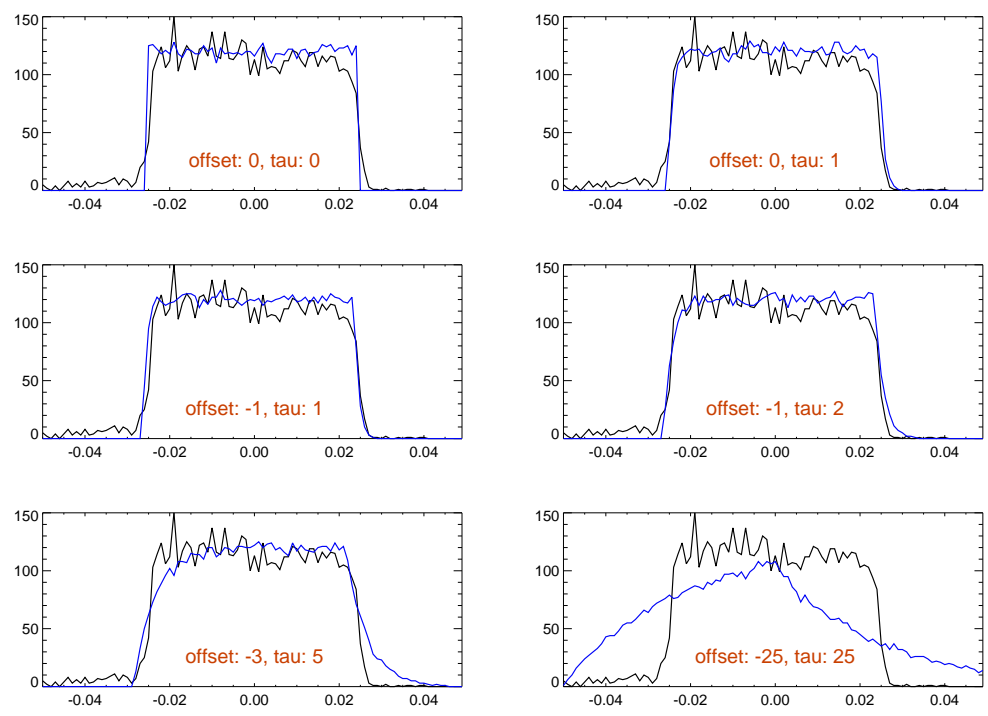

Figure 5: Monte-Carlo simulations vs. observation. Black: ACS time-lag distribution, same as in the previous plot. Blue: Monte-Carlo Simulation.

The best parameter combination, off set $=-1$ and $t a u=1$, indicates that both the ACS clock and the SPI germanium detector clock are stable. The relative drift $\leq 1 \mathrm{~ms}$. It cab also be inferred that the last germanium detector event before the saturation is a good indication of the onset of the saturation. 


\section{Result and Conclusion}

The drift of ACS clock with respect to the germanium detector clock during the whole INTEGRAL mission is around $1 \mathrm{~ms}$. The small broadening of the time lag distribution indicates that the last photon recorded by the germanium detectors is a good indication of the onset of the saturation, and is probably an event generated by the incident energetic cosmic-ray. This value is much smaller than the previous reported $10 \mathrm{~ms}$ [7].

\section{Acknowledgements}

ZXL is grateful for DLR support (contract No. FKZ 50 OG 0502).

\section{References}

[1] von Kienlin, A., et al. 2003, A\&A, 411, L299

[2] Lichti, G. G., Georgii, R., von Kienlin, A., Schönfelder, V., Wunderer, C., Jung, H.-J.,

[3] Hurley, K., INTEGRAL in the Third Interplanetary Network for Gamma-Ray Bursts, in Proc. 4th INTEGRAL Workshop, Exploring the Gamma-Ray Universe, ESA SP 459, p. 391, 2001

[4] Hurley, K., von Kienlin, A., Lichti, G., and Rau, A., INTEGRAL Joins the 3rd Interplanetary Network, in proc. 5th INTEGRAL Workshop, The INTEGRAL Universe, ESA SP 552, p. 645, 2004

[5] Hurley, K., Rau, A., von Kienlin, A., and Zhang, X.-L., this workshop

[6] Rau, A., Kienlin, A. V., Hurley, K., \& Lichti, G. G. 2005, A\&A, 438, 1175

[7] Rau, A., von Kienlin, A., Lichti, G., Hurley, K., \& Beck, M. 2004, GRB Coordinates Network, 2568, 1 